\title{
Alexis Levrier, Les journaux de Marivaux et le monde des «spectateurs»
}

\section{Franco Piva}

\section{(2) OpenEdition}

1 Journals

\section{Edizione digitale}

URL: http://journals.openedition.org/studifrancesi/8260

DOI: 10.4000/studifrancesi.8260

ISSN: 2421-5856

\section{Editore}

Rosenberg \& Sellier

\section{Edizione cartacea}

Data di pubblicazione: 1 mai 2009

Paginazione: 173-174

ISSN: 0039-2944

\section{Notizia bibliografica digitale}

Franco Piva, «Alexis Levrier, Les journaux de Marivaux et le monde des «spectateurs»», Studi Francesi [Online], 157 (LIII | I) | 2009, online dal 30 novembre 2015, consultato il 08 janvier 2021. URL: http:// journals.openedition.org/studifrancesi/8260 ; DOI: https://doi.org/10.4000/studifrancesi.8260

Questo documento è stato generato automaticamente il 8 janvier 2021.

\section{(c) (i) $\odot$}

Studi Francesi è distribuita con Licenza Creative Commons Attribuzione - Non commerciale - Non opere derivate 4.0 Internazionale. 


\title{
Alexis Levrier, Les journaux de Marivaux et le monde des «spectateurs»
}

\author{
Franco Piva
}

\section{NOTIZIA}

ALEXIS LEVRIER, Les journaux de Marivaux et le monde des «spectateurs». Préface de Françoise GEVREY, Paris, Presses de l'Université Paris-Sorbonne, 2007, pp. 521.

1 Era da tempo noto il grande successo, commerciale e di pubblico, che lo Spectator, il "foglio" pubblicato dall'inizio del 1711 alla fine del 1712 dagli inglesi Joseph Addison et Richard Steele conobbe nel Paese che lo vide nascere, così come era noto il grande impatto che quel giornale ebbe sul Continente, dove esso fu presto tradotto ed imitato. A questo proposito, il nome che veniva fatto più spesso era quello di Marivaux, il cui Spectateur français è stato a lungo considerato come una più o meno fedele imitazione dello Spectator inglese. Certo, di tanto in tanto si faceva anche qualche altro nome e si citava qualche altro titolo, ma per lo più en passant e senza porre troppa attenzione ad un fenomeno che era visto prima di tutto come un fatto di origine e natura essenzialmente economico: lo sfruttamento, da parte di plumitifs più o meno a corto di ispirazione, di una formula che, al seguito del successo incontrato dal giornale di Addison e di Steele, portasse loro qualche facile risorsa. In fondo, anche lo Spectateur français fu visto a lungo in questa ottica. Il panorama è venuto mutando in questi ultimi decenni grazie ad una serie di lavori che sono culminati nella thèse di Michel Gilot (Les journaux de Marivaux. Itinéraire moral et accomplissement esthétique, 1974) che, fondamentale in sé, non ha avuto però la diffusione che meritava, e che è rimasta ad ogni modo incentrata quasi esclusivamente su Marivaux. Certo, Gilot lasciava intravedere un mondo ben più vasto ma le indicazioni che la sua thèse dava al riguardo erano troppo sommarie perché il lettore potesse avere la percezione quantitativa e 
qualitativa di quell'“altro" su cui Gilot aveva gettato uno sguardo ahimé troppo frettoloso.

2 È proprio quell' "altro" che Alexis Lévrier ha inteso prendere in considerazione nella sua thèse: la quale, se continua ad avere al centro Marivaux, come il titolo suggerisce, ed in particolare il Marivaux giornalista, attorno a quel nome costruisce un vero e proprio mondo che, partendo da Addison e da Steele, si raccoglie attorno a quello che Lévrier chiama il «phénomène spectatorial»; un fenomeno assai più vasto di quanto potesse credersi e che il giovane studioso d'oltralpe ha ricostruito con molta cura ed intelligenza, partendo dallo Spectator di Addison e di Steele per arrivare fino al primo essoufflement che il fenomeno ha conosciuto, in terra francese, con la fine del Cabinet $d u$ philosophe, l'ultimo "foglio" di Marivaux, nel 1734. Il periodo preso in considerazione ha consentito a Lévrier di costituire un corpus di una dozzina di "fogli" che, in forme e proporzioni diverse, si inseriscono nel "phénomène spectatorial» che lo studioso esamina da vicino partendo dal "foglio" capostipite, quello lanciato da Addison e da Steele.

3 Il lavoro di Alexis Lévrier si articola essenzialmente in quattro parti: la prima, partendo dal "foglio" di Addison e di Steele, definisce gli elementi che caratterizzano il fenomeno, così come esso è stato lanciato e praticato dai due giornalisti inglesi: periodicità intensa quanto regolare, stretto rapporto col pubblico, ruolo di guida morale e comportamentale che questo rapporto consente, anche per l'importanza e l'imponenza che la figura dello "Spectator" assume fin dall'inizio nei riguardi del lettore, ecc. In un secondo momento Lévrier prende in considerazione le prime imitazioni che lo Spectator ha suscitato in Olanda negli anni immediatamente successivi al lancio del giornale Oltremanica o comunque nel decennio 1712-1720: dapprima con il Misanthrope di Justus Van Effen, poi con il Censeur di Rousset de Missy, infine con La Bagatelle ancora di Van Effen. Fogli che, pur adottando nomi diversi, seguono abbastanza da vicino il modello inglese.

La terza tappa è rappresentata dallo Spectateur français di Marivaux, i cui primi numeri apparvero nel 1721, e, in misura minore, dagli altri due suoi "fogli": L'Indigent philosophe e il Cabinet du philosophe. Con Marivaux il rapporto col modello inglese, seppur evidente, si fa decisamente diverso: l'imitazione si fa assai più libera, anche, se non soprattutto, per l'adozione dello strumento, tipicamente francese, della «feuille volante» che comporta, già di per sé un rapporto con il lettore molto diverso da quello adottato dai due giornalisti inglesi; un rapporto che è però da mettere anche in relazione con la figura dello «spectateur français», assai più distaccato, disincantato e, perciò, anche assai meno affidabile, come guida, dello "Spectator" inglese, oltre che per la natura stessa della sua osservazione, molto più estemporanea, istintiva e legata al piacere del momento più di quanto non lo fosse quella del serioso "Spectator" messo in scena da Addison e da Steele, con conseguenze evidenti anche sulla scrittura, molto più frammentata e «capricieuse», come la definisce Lévrier.

Di queste trasformazioni risentirono gli Spectateurs che furono pubblicati in Francia nel decennio che seguì il lancio dello Spectateur français di Marivaux. Si tratta di cinque o sei tentativi che incontrarono fortune diverse e che con lo Spectateur di Marivaux intrattennero rapporti diversi ancorché evidenti, come dimostra il loro titolo. Tra questi tentativi uno in particolare ci pare meriti l'attenzione dello studioso moderno: quello dell'anonimo autore della Spectatrice: non solo perché introduce un punto di vista diverso rispetto agli altri "spectateurs" ma anche perché, nei riguardi dello 
Spectateur marivaudiano, come peraltro nei riguardi dello Spectator inglese, si pone in una posizione volutamente più ambigua se non più distaccata.

Dalla ricostruzione puntuale ed intelligente di Alexis Lévrier, che avrebbe avuto bisogno di assai più spazio per essere presentata nella sua articolata complessità, esce un quadro di una grande ricchezza ed un fenomeno, quello degli «spectateurs» che se, come osserva lo stesso Lévrier, non dette origine ad un genere letterario vero e proprio, non fosse che per la diversità delle varie esperienze di scrittura che si possono ricondurre all'interno del fenomeno preso in esame, costituisce tuttavia un momento importante della letteratura, e più in generale della cultura francese del Settecento, che merita per ciò stesso, da parte degli studiosi, un'attenzione assai maggiore di quella che ad esso è stata da loro riservata sino ad ora. 